\title{
ESTUDIO ELECTROQUÍMICO DEL ACERO AISI-SAE 1020, NITRURADO VÍA PLASMA DURANTE TIEMPOS DE 10 Y 15 HORAS
}

Por:

Nelson Tovar ${ }^{1}$, Ubeimar Fuentes' ${ }^{1}$ Cesar Ortiz ${ }^{1}$, Enrique Vera ${ }^{1}$

\section{RESUMEN}

En la presente investigación se utilizó como sustrato el acero AISI-SAE 1020, al cual se realizó nitruración por plasma durante 10 y 15 horas continuas. La diferencia de potencial suministrada fue entre 400 y 500 V. En una campana de 6.11 litros de volumen se mantuvo un flujo constante de nitrógeno puro de $0.5 \mathrm{ml} /$ $\mathrm{s}$, la presión de trabajo fue de 3 torr aproximadamente. Se obtuvo una temperatura final de tratamiento de $82{ }^{\circ} \mathrm{C}$ para el sustrato nitrurado durante 10 horas $y$, de $88{ }^{\circ} \mathrm{C}$ para el de 15 horas; estas temperaturas fueron obtenidas sin calentamiento auxiliar. Con un equipo potenciostato-galvanostato GAMRY, se realizaron pruebas electroquímicas de EIS y Tafel; dichas técnicas permitieron observar que la nitruración por plasma mejora la resistencia a la corrosión del acero nitrurado. Así mismo, mediante un microdurometro, HVS-1000 se realizaron medidas de microdureza de las superficies tratadas, donde se obtuvo un aumento en la dureza del material nitrurado.

Palabras Clave: Electroquímica, Nitruración.

\section{ABSTRACT}

In the present investigation was nitrided the steel AISI-SAE 1020 by means of the process plasma nitriding during 10 and 15 hours continuous. The potential difference applied between 400 and 500 $V$. The nitrogen flow was $0.5 \mathrm{ml} / \mathrm{s}$ constant. The final temperature of treatment was $82{ }^{\circ} \mathrm{C}$ for the substratum nitrided for 10 hours and of $88^{\circ} \mathrm{C}$ for 15 hours; these temperatures were obtained without auxiliary warming. With a potentiostaticgalvanostatic tester GAMRY, was ejecuted the technical electrochemical EIS and Tafel, the plasma nitriding improved the corrosion resistance of the steel nitrided. A equip of micro hardness tester HVS-1000, showed an increase on the hardness of the material nitrured.

Key words: Electrochemical, Nitriding.

\section{INTRODUCCIÓN}

La corrosión es un fenómeno según el cual los metales tienen tendencia, bajo la acción de agentes atmosféricos o de reactivos químicos, a volver a su estado original de óxido u otros que son más estables con relación al medio considerado [2]. La nitruración ha sido usada para mejorar el desempeño de los materiales frente al desgaste. Sin embargo, también puede mejorar o disminuir la resistencia a la corrosión, dependiendo del material y del medio ambiente empleado [4].

\section{DESARROLLO EXPERIMENTAL}

Se pulieron 12 probetas circulares de $11 \mathrm{~mm}$ de radio y $2 \mathrm{~mm}$ de espesor, de acero AISI-SAE 1020, hasta obtener brillo metalográfico; esto se logra mediante pulido consecutivo con papel de lija 80, 300, 600 y 1000 , por último se brilla el sustrato mediante pulido con paño y alúmina. Este proceso se repitió para cada una de las probetas [1].

Figura 1. Nitruración iónica durante: a) Blanco b) 10 horas c) 15 horas.
a)
b)
c)
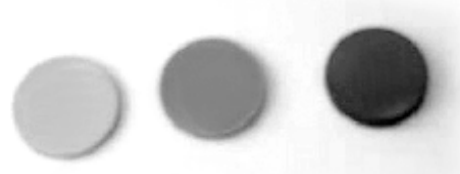

'Universidad Pedagógica y Tecnológica de Colombia, Grupo de Superficies, Electroquímica y Corrosión GSEC, nelsonuriel@yahoo.com, ubefuentes@yahoo.es, cesaroti@tunja.uptc.edu.co,grupo_gsec@yahoo.es 
Estudio electroquímico del acero AISI-SAE 1020, nitrurado vía plasma durante tiempos de 10 y 15 horas

\section{CONDICIONES DE NITRURADO}

Entre dos electrodos de placas circulares paralelas, se aplicó una diferencia de potencial entre 400 y $500 \mathrm{~V}$. Mediante una bomba mecánica previamente se hace vacío, en una campana de vidrio de 6.11 litros de volumen, hasta 1 torr de presión luego de lo cual se permite el flujo de nitrógeno puro al interior de la cámara; el voltaje aplicado y el flujo de gas deben ajustarse de tal manera que permita al plasma alcanzar la estabilidad. Dicha estabilidad se obtuvo a una presión de trabajo de 3 torr aproximadamente, un flujo de $0.5 \mathrm{ml} / \mathrm{s}$, y un potencial entre placas de $466 \mathrm{~V}$. La temperatura final de tratamiento para las probetas nitruradas fue de $82{ }^{\circ} \mathrm{C}$ para un tiempo de 10 horas, y de $88^{\circ} \mathrm{C}$ para el sustrato nitrurado durante 15 horas.

\section{PRUEBAS ELECTROQUÍMICAS}

Se realizaron las pruebas electroquímicas de EIS y Tafel en un equipo Potenciostato Galvanostato marca GAMRY, perteneciente al grupo de investigación GSEC, mediante una celda electroquímica apropiada. Se efectuaron pruebas electroquímicas en sustrato nitrurado vía plasma durante 10 y 15 horas, así como en una probeta sin nitrurar (blanco). Como electrodo de referencia se usó el electrodo de Plata-cloruro de plata $(\mathrm{Ag} / \mathrm{AgCl})$ y como electrolito una solución de $\mathrm{NaCl}$ al $1 \%$ con un $\mathrm{PH}$ de 5.4 .

\section{ANALISIS DE RESULTADOS}

La prueba EIS se realizó en un rango de frecuencias entre $10 \mathrm{mHz}$ y $100 \mathrm{KHz}$ para todas las probetas. En el diagrama del ángulo de fase (figura 2) se observa que para cada una de las probetas analizadas, el ángulo de fase para altas y bajas frecuencias es de 0 grados y el único domo observado es propio de la impedancia capacitiva de la doble capa eléctrica. Se observa también que el ángulo de desfase aumenta a medida que se eleva el tiempo de nitrurado, esto es debido posiblemente al aumento en el espesor de la zona nitrurada.

Figura 2. Diagrama del ángulo de fase

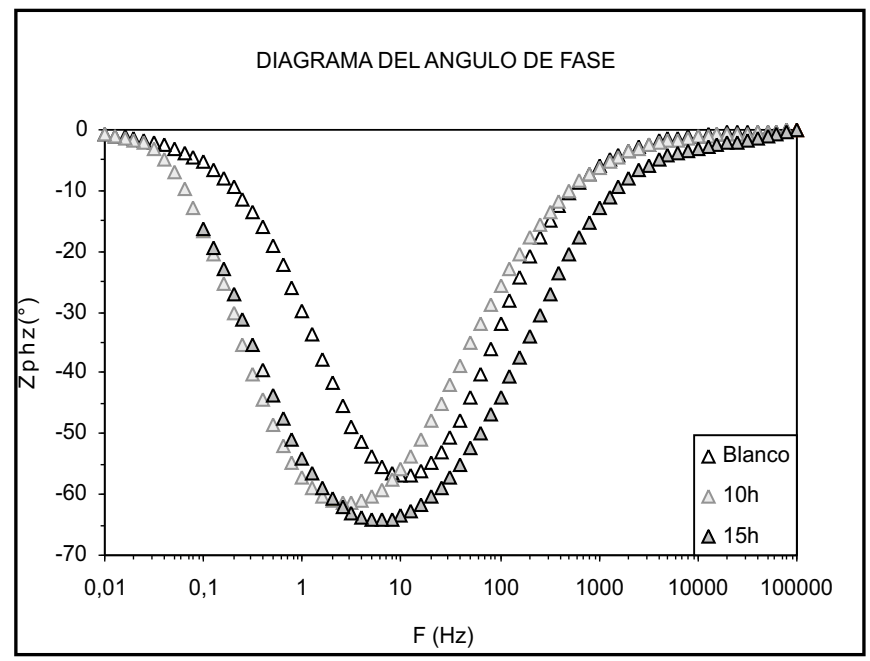

Figura 3. Diagrama de Niquist

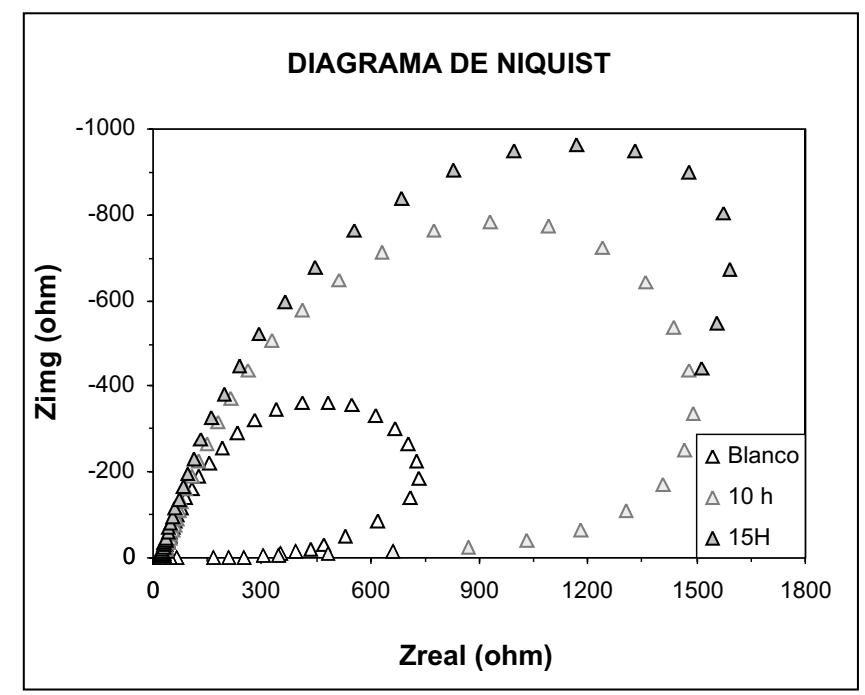

La no existencia de un segundo domo en los diagramas de fase y Niquist (Figuras 2 y 3), significa que no existe una capa de recubrimiento sobre el sustrato. Lo anterior confirma que la nitruración iónica no es un proceso de recubrimiento, sino una modificación de la superficie del material tratado. 
Estudio electroquímico del acero AISI-SAE 1020, nitrurado vía plasma durante tiempos de 10 y 15 horas

Figura 4. Diagrama de impedancia

\section{DAGRAMA DE IM PEDANCIA}

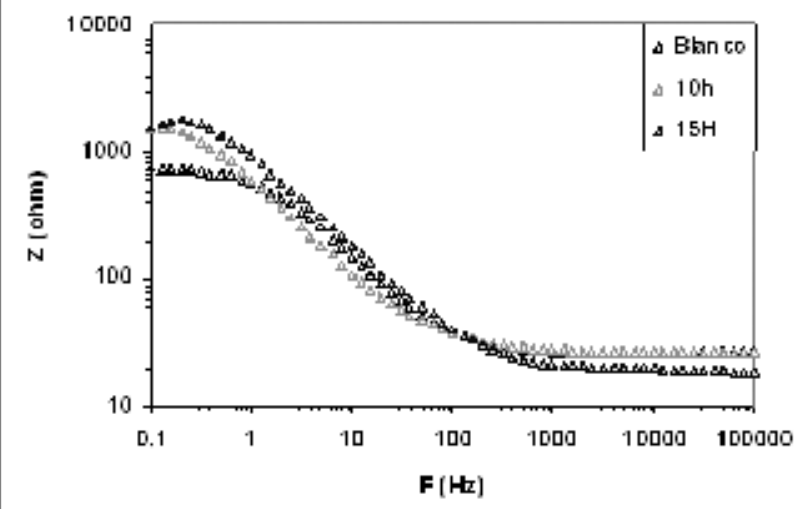

Tabla 1. Resultados de la prueba electroquímica EIS

\begin{tabular}{|c|c|c|c|c|}
\hline $\begin{array}{c}\text { Tiempo de } \\
\text { nitruración }\end{array}$ & $\operatorname{Rs}($ ) & $\operatorname{Rp}($ ) & CPE ( F) & $n$ \\
\hline Blanco & 26.71 & 760.2 & 200 & 0.85 \\
\hline 10 horas & 26.53 & 1540 & 400 & 0.82 \\
\hline 15 horas & 18.45 & 1767 & 150 & 0.85 \\
\hline
\end{tabular}

La figura 4. muestra que la impedancia de la interfase, metal-electrolito, aumenta con el tiempo de nitrurado del material base. Se puede afirmar, entonces, que la nitruración iónica produce en el material comportamientos resistivos importantes cuyos valores se muestran en la tabla 1. Los sistemas nitrurados en esta investigación presentan el mismo circuito equivalente (figura 5).

Figura 5. Circuito equivalente para el sustrato nitrurado

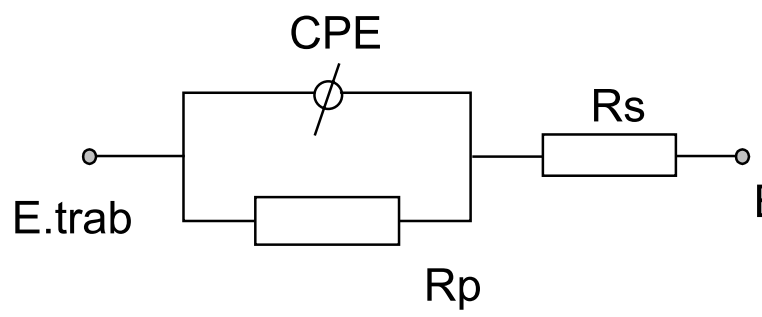

Se observa que la resistencia de polarización de la probeta nitrurada durante 10 horas, es el doble de la correspondiente al sustrato base y, además, muy cercana a la impedancia obtenida al nitrurar durante 15 horas. Esto muestra que la nitruración iónica produce superficies resistentes contra la corrosión, la cual mejora al elevar el tiempo de nitrurado.

\section{PRUEBA DE TAFEL}

El análisis de las probetas se realizó en un rango de barrido entre -0.2 y $+0.2 \mathrm{~V}$ con respecto al potencial de corrosión Eoc, la velocidad de escaneo fue de $1 \mathrm{mV} / \mathrm{s}$ [2]. Los resultados obtenidos se muestran el la tabla 2.

Figura 6. Diagrama de Tafel.

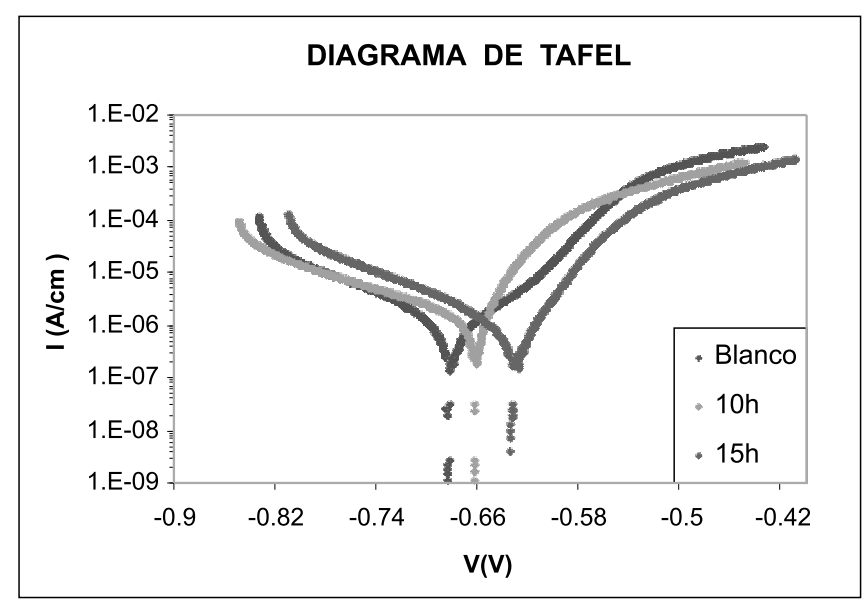

Tabla 2. Resultados de la prueba electroquímica Tafel.

\begin{tabular}{|c|c|c|}
\hline $\begin{array}{c}\text { Tiempo de } \\
\text { tratamiento }\end{array}$ & Eoc $(\mathrm{mV})$ & Vel corr $(\mathrm{mpy})$ \\
\hline Blanco & -684 & 0.2636 \\
\hline 10 horas & -663 & 0.2698 \\
\hline 15 horas & -634 & 0.1711 \\
\hline
\end{tabular}

Los resultados suministrados por el diagrama de Tafel (figura 6.) muestran que la velocidad de corrosión no cambia significativamente, lo que se observa es que el potencial de corrosión Eoc, se hace más positivo al aumentar el tiempo de nitrurado. Esto es debido, 
Estudio electroquímico del acero AISI-SAE 1020, nitrurado vía plasma durante tiempos de 10 y 15 horas

posiblemente a la presencia de nitruros de hierro en la superficie del material tratado y/o la presencia de nitrógeno implantado en la estructura del material base.

\section{PRUEBA DE MICRODUREZA}

Esta prueba fue realizada con un microdurómetro HVS-1000, perteneciente al laboratorio del grupo GSEC de la UPTC. Se realizaron 5 indentaciones sobre el sustrato nitrurado, sin cortar la pieza, así como en una probeta sin nitrurar. Los resultados obtenidos se encuentran explícitos en la tabla 3.

El análisis de esta prueba muestra que la microdureza de las probetas nitruradas no tiene un aumento significativo cuando se hace mayor el tiempo de tratamiento, con respecto al material base. Lo anterior se puede observar con claridad en la figura 7.

Figura 7. Diagrama de Dureza HV.

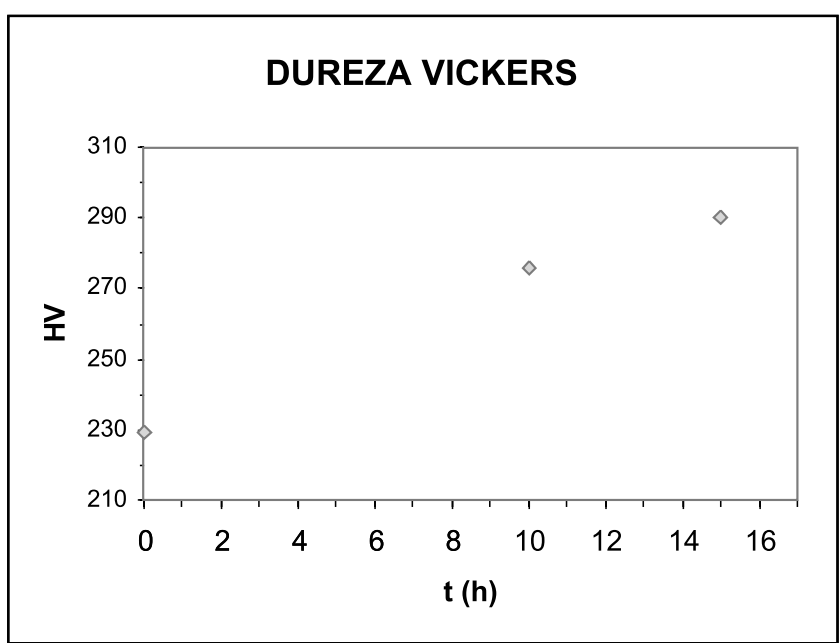

Tabla 3. Resultados prueba de Microdureza.

\begin{tabular}{|c|c|}
\hline Tiempo $(\mathrm{h})$ & Dureza Vickers $(\mathrm{HV})$ \\
\hline 0 & 229 \\
\hline 10 & 276 \\
\hline 15 & 290 \\
\hline
\end{tabular}

\section{ESPESOR}

Los resultados de la tabla 4. evidencian el aumento de espesor de la zona de difusión cuando se eleva el tiempo de tratamiento. El margen de error depende, en su mayoría, de la uniformidad de la zona modificada (figura 8 ).

Figura 8. Zonas de nitruración en sustrato tratado durante 15 horas. Fotografía a 400X.

Material base

Zona de difusión

Zona de compuestos

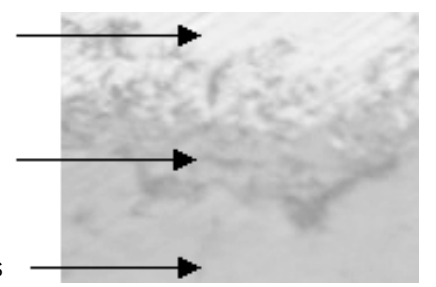

Tabla 4. Espesor aproximado de la zona de difusión.

\begin{tabular}{|c|c|}
\hline Nitruración & Espesor $(\mu \mathrm{m})$ \\
\hline 10 horas & $6.5 \pm 1.4$ \\
\hline 15 horas & $7.1 \pm 1$ \\
\hline
\end{tabular}

\section{ANÁLISIS QUÍMICO}

Se realizó un análisis de DRX en probetas nitruradas a 10 y 15 horas, en los que se obtuvo espectros iguales. El espectro se muestra en la figura 9. En él se puede observar que no se encontraron compuestos de nitruros de hierro en las probetas tratadas. Esto sucedió posiblemente a que las temperaturas obtenidas al final del proceso fueron muy bajas debido al no uso de calentamiento auxiliar durante la nitruración. Los grandes picos mostrados correspondientes al $\mathrm{Fe}$ y $\mathrm{Fe}_{3} \mathrm{C}$. Así mismo se efectuó un análisis químico mediante SEM, en donde tampoco se encontró la presencia de nitrógeno en el material tratado [3]. 
Estudio electroquímico del acero AISI-SAE 1020, nitrurado vía plasma durante tiempos de 10 y 15 horas

Figura 9. Espectro DRX obtenido para 10 y 15 horas de nitruración.

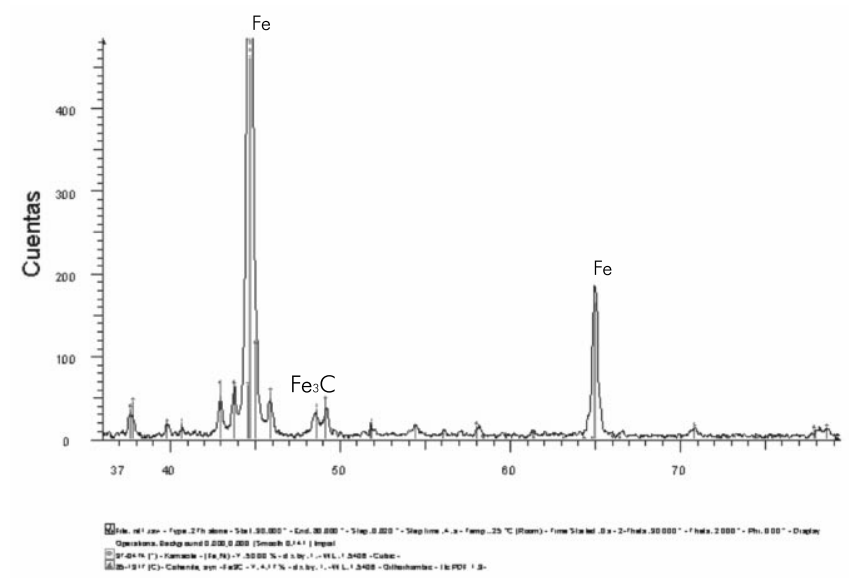

ANÁLISIS METALOGRÁFICO

Debido a que no se obtuvieron los resultados esperados en los análisis realizados con DRX y SEM, se optó por realizar un análisis metalográfico con Nital al $5 \%$ en volumen. Las superficies nitruradas a 10 y 15 horas fueron atacadas durante un minuto.

Figura 10. Nitruración durante 15 horas Fotografía a $100 x$.

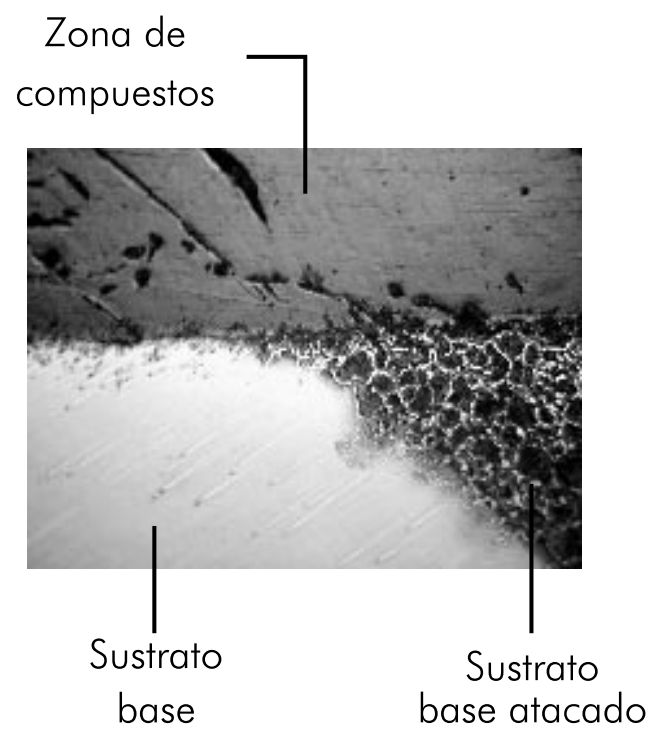

Figura 11. Nitruración durante 10 horas Fotografía a $400 X$.

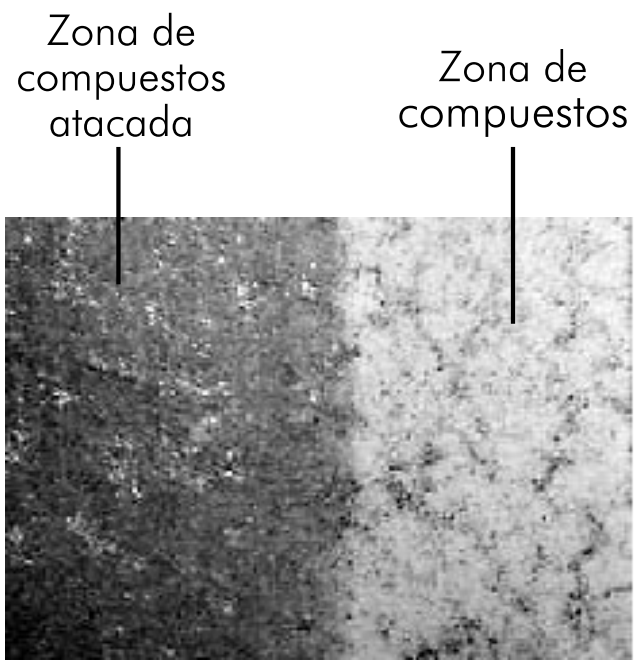

La figura 10. muestra que la zona nitrurada no se ve afectada por la presencia del reactivo, como sí se observa en el material base, donde se revela la estructura martensítica (negro) y ferrita (café). El hecho de que la zona modificada no presente alteración con el ataque químico, indica que se obtuvo una muy buena superficie nitrurada, como protectora del material base [5].

En la figura 11. la zona nitrurada durante 10 horas (derecha), presenta un cambio en la coloración al ser atacada con el reactivo revelador (izquierda). Lo anterior puede ser atribuido al hecho de que esta probeta posee un menor espesor en la superficie tratada, por cuanto fue nitrurada durante un tiempo menor que en el caso anterior.

\section{CONCLUSIONES}

Se obtuvo buenos resultados en el estudio de impedancia electroquímica, ya que la resistencia de polarización aumenta al doble de la obtenida en el sustrato blanco.

El diagrama de Niquist y el diagrama de fase demuestran que la nitruración iónica no es un proceso de 
Estudio electroquímico del acero AISI-SAE 1020, nitrurado vía plasma durante tiempos de 10 y 15 horas

recubrimiento del material, sino una modificación de la superficie tratada, puesto que en ambos se muestran la formación de un solo domo, lo cual es característico de la impedancia capacitiva existente en la doble capa electrizada.

La prueba de Tafel permite determinar que la nitruración iónica no disminuye la velocidad de corrosión del material, lo que se observa es que el potencial de corrosión se hace más positivo cuando se eleva el tiempo de nitrurado.

La dureza acero AISI-SAE 1020 no aumenta significativamente con el tiempo de tratamiento de nitrurado, respecto del sustrato blanco.

El ataque realizado con nital, permitió establecer que se obtuvo una buena capa de compuestos, dado que no se vio modificada con el ataque del reactivo utilizado.

\section{BIBLIOGRAFIA}

[1] PINO, Alfredo; LÓPEZ, Alfonso. Nitruración por plasma. Trabajo de grado. UPTC. Tunja. 1977. 150p

[2] MANSFELD, Florian. Analysis and interpretation de EIS data for metals and alloys. Technical report 26. Solartron. USA, 1993.

[3] RAMIREZ C., Miguel. Nitruración de acero 1018 con descarga eléctrica de resplandor. UAM - A. Proyecto terminal. Azcapotzalco. México. 2000. Pág. 1-19.

[4] CORRENGIA, P., et all. Comportamiento frente a la corrosión de aceros nitrurados por plasma. Centro de Investigación y Desarrollo en Mecánica (CEMEC). 2002.

[5] BRÜHL, Sonia. Procesamiento de materiales por plasma. Universidad Tecnológica Nacional.
Apunte para el curso dictado en marzo de 2003. Uruguay.

Recibido : Septiembre 1 de 2006

Aceptado: Diciembre 10 de 2006 